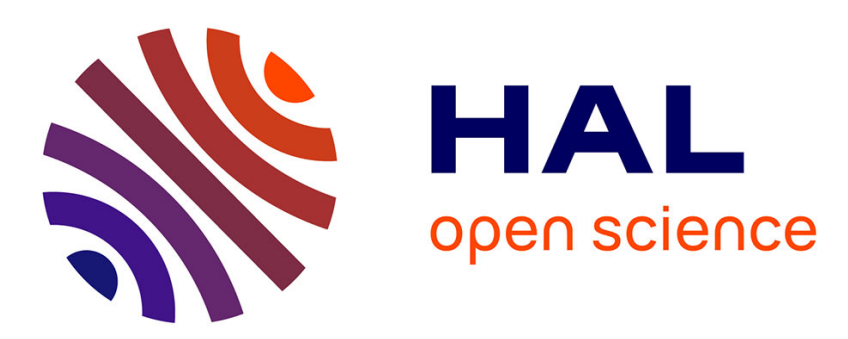

\title{
Réponses de la production et de la composition du lait de vache aux variations d'apports nutritifs
}

\author{
J.B. Coulon, B. Remond
}

\section{To cite this version:}

J.B. Coulon, B. Remond. Réponses de la production et de la composition du lait de vache aux variations d'apports nutritifs. Productions Animales, 1991, 4 (1), pp.49-56. hal-00895924

\section{HAL Id: hal-00895924 \\ https://hal.science/hal-00895924}

Submitted on 1 Jan 1991

HAL is a multi-disciplinary open access archive for the deposit and dissemination of scientific research documents, whether they are published or not. The documents may come from teaching and research institutions in France or abroad, or from public or private research centers.
L'archive ouverte pluridisciplinaire HAL, est destinée au dépôt et à la diffusion de documents scientifiques de niveau recherche, publiés ou non, émanant des établissements d'enseignement et de recherche français ou étrangers, des laboratoires publics ou privés. 
INRA Prod. Anim., 1991, 4 (1), 49 - 56

\section{J.B. COULON et B. REMOND}

INRA Laboratoire de la Lactation et de l'Elevage des Ruminants Theix - 63122 Saint-Genès-Champanelle
Réponses de la production et de la composition du lait de vache aux variations d'apports nutritifs

\begin{abstract}
La production et la composition du lait varient en fonction des apports nutritifs, en particulier énergétiques. Mais quelle est la forme de ces réponses et sont-elles différentes selon le stade de lactation et le potentiel de production des animaux?
\end{abstract}

\begin{abstract}
Une augmentation du niveau d'alimentation énergétique des vaches laitières, très généralement réalisée par une distribution plus libérale d'aliment concentré, entraîne une augmentation de production laitière, qui suit la loi des rendements décroissants (Wiktorsson 1979, Gordon 1980, Broster et Thomas 1981, Rémond 1985, Leaver 1988). La diminution progressive de l'efficacité marginale des apports alimentaires a 2 causes principales : 1) Un accroissement de plus en plus faible de la quantité d'énergie réellement mise à la disposition des animaux, au fur et à mesure que l'apport de concentré s'accroît. Ce ralentissement est lié d'une part à la diminution de plus en plus
\end{abstract}

\begin{abstract}
Résumé
L'objectif de cette étude bibliographique a été de préciser l'influence du stade de lactation, de l'âge, du niveau de production des animaux et de la durée de la sous(sur)-alimentation, sur la réponse de la production laitière et du taux protéique à des apports variables d'énergie. Soixante-six essais d'alimentation réalisés sur des vaches laitières produisant entre 9 et $29 \mathrm{~kg} / \mathrm{j}$ ( 6 à 30 vaches/lot expérimental) et comportant au moins 3 niveaux d'apports énergétiques ont été utilisés. Ces apports énergétiques ont été exprimés en énergie réellement disponible pour l'animal (énergie nette de lactation, UFL). En début de lactation, la réponse de production laitière aux apports énergétiques a été linéaire et élevée $(+0,9 \mathrm{~kg}$ de lait/UFL). En milieu de lactation et dans les essais de longue durée, cette réponse a été curvilinéaire. Lorsque les apports étaient inférieurs aux besoins, elle a été plus forte dans les essais de longue durée que dans ceux réalisés sur de courtes périodes en milieu de lactation $(2,2 \mathrm{~kg} / \mathrm{UFL}$ contre $1,3 \mathrm{~kg} / \mathrm{UFL}, P<0,01)$. La réponse du taux protéique aux apports énergétiques a été linéaire quelles que soient la période et la durée des essais. Elle a été 2 fois plus faible en début de lactation (0,3 g p.1000/UFL) qu'en milieu de lactation ou qu'au cours des essais de longue durée $(0,6 \mathrm{~g}$ p.1000/UFL). La relation entre les variations de la production laitière et du taux protéique a été curvilinéaire et a dépendu du stade de lactation et du niveau de couverture des besoins énergétiques. Il n'a pas été possible de mettre en évidence d'effet important du niveau de production des animaux sur les réponses de la production laitière ou du taux protéique aux variations d'apports énergétiques.
\end{abstract}

importante de la quantité de fourrage ingérée lorsqu'il est distribué à volonté (phénomène de substitution, Dulphy et al 1987), et d'autre part à l'accroissement de plus en plus faible de la digestibilité de la ration par suite de l'augmentation du niveau d'ingestion et de la proportion de l'aliment concentré dans la ration (Doreau et Rémond 1983, Vermorel et al 1987), 2) Une proportion décroissante de l'énergie supplémentaire réellement mise à la disposition de la mamelle. En effet, au fur et à mesure que la capacité maximale de production laitière est approchée, une part croissante de l'énergie disponible pour la production est déposée dans l'organisme de l'animal, essentiellement sous forme de lipides (Broster et Broster 1984). Inversement, pour des apports inférieurs à la couverture stricte des besoins, une partie de l'énergie utilisée pour la production laitière provient des réserves corporelles.

Ces modifications de la digestion et de l'utilisation métabolique de l'énergie liées à des changements du niveau d'alimentation sont sujettes à des variations importantes d'un essai à l'autre (Gordon 1980). Différentes causes de cette variabilité ont déjà été mises en évidence ou suggérées. Elles relèvent soit des caractéristiques des animaux : stade physiologique (Broster et al 1985) ; état corporel (Larnicol 1984, Garnsworthy 1988, Jones et Garnsworthy 1989 ) ; capacité de production laitière (Wiktorsson 1971 et 1979, Johnson 1979, Gordon 1984, Coulon et al 1987), soit de leur nutrition autre qu'énergétique et en particulier de la satisfaction des besoins azotés. Il a en effet été montré (Rémond 1985, Vérité 1989) que la réponse de production laitière aux apports alimentaires azotés suivait également la loi des rendements décroissants. Enfin, la durée d'expérimentation peut aussi être un facteur de variation de la réponse de la production laitière aux apports énergétiques. 
L'influence des apports énergétiques sur la teneur en protéines du lait est sujette aux mêmes variations. Elle a cependant fait l'objet de travaux de synthèse beaucoup moins nombreux et complets (Emery 1978, Rémond 1985, Sutton 1989, Spörndly 1989).

L'objectif de cette étude est de préciser la courbe de réponse de la quantité de lait produite et de ses teneurs en matières grasses et en protéines à des apports variables d'énergie, selon les caractéristiques des animaux et de leur alimentation, ainsi que selon la durée d'expérimentation. Elle a été réalisée avec des résultats bibliographiques, à partir desquels les variations d'apports énergétiques ont été exprimées dans le système d'énergie nette le plus récent et donc a priori le plus précis (Vermorel 1989).

\section{1 / Données utilisées}

\section{1 / Origine et caractéristiques des données}

Soixante-six essais d'alimentation, français (13) et étrangers (53) dans lesquels au moins 3 niveaux d'apports énergétiques (soit 216 lots expérimentaux] étaient comparés, ont été utilisés. Leurs caractéristiques détaillées sont précisées dans les tableaux 1 et 2 . Dans chacun de ces essais, un des niveaux d'apport pouvait être considéré comme proche des recommandations proposées par Faverdin et al (1987) : pour les

\section{Tableau 1. Description des essais utilisés.}

Douze des 66 essais utilisés avaient été conduits en début de lactation (8 à 13 premières semaines, essais D), 33 en milieu de lactation (après le pic de production) sur une durée de 4 à 11 semaines (essais M), et 21 sur une durée de 18 à 40 semaines (essais L). Les lots expérimentaux étaient constitués de 6 à 30 vaches (primipares dans 13 essais; multipares dans les autres) selon les essais. Le niveau de production des lots d'animaux a varié entre 9 et $29 \mathrm{~kg} / \mathrm{j}$ de lait à $4 \%$ de matières grasses (lait $4 \%$ ). Les rations utilisées étaient à base de foin (23), d'ensilage d'herbe (37) ou d'ensilage de maïs (6), très généralement offerts à volonté (51 essais). Elles étaient complémentées avec des aliments concentrés, composés dans la majorité des cas d'un mélange de céréales et de tourteaux, dont la proportion était comprise entre 0 et $80 \%$ (40\% en moyenne) de la ration totale sur la base de la matière sèche (MS).

Dans tous les essais, les quantités d'aliments ingérées et de lait produites avaient été mesurées individuellement. Dans 53 d'entre eux (soit 172 lots expérimentaux), on disposait aussi du taux protéique (ou d'azote) du lait ou (pour 10 essais) de la teneur en MS délipidée du lait, dont les variations ont été assimilées à celles du taux protéique. essais en milieu de lactation (M) et de longue durée (L), l'écart entre les besoins et les apports corrigés était en moyenne faible, de même que les variations de poids vif, lorsqu'elles étaient disponibles (tableau 2). Dans les essais réalisés en début de lactation (D), le déficit énergétique observé dans le lot témoin était en moyenne comparable à celui proposé dans les dernières recommandations françaises. Dans la plupart des essais, les besoins azotés des animaux étaient constamment couverts. Seuls 26 lots expérimentaux (conduits en milieu de lactation) ont reçu des rations avec des teneurs en MAT faibles $(<13 \%)$ et croissant avec le niveau des apports énergétiques d'un lot à l'autre dans le même essai ; ils ont été analysés séparément.

Sur l'ensemble des lots expérimentaux initialement disponibles, 7 n'ont pas été utilisés pour l'analyse des variations de la production laitière, et 5 pour l'analyse des variations du taux protéique. Leurs résultats se situaient très largement en dehors de l'évolution générale observée (écart entre la valeur réelle et la valeur estimée supérieure à 2 écart-types).

\section{2 / Analyse des données}

Pour chaque lot expérimental de chaque essai, les apports et les besoins énergétiques ont été calculés en Unités Fourragères Lait $(1 \mathrm{UFL}=7,1 \mathrm{MJ}$ d'énergie nette $=11,7 \mathrm{MJ}$ d'énergie métabolisable) (Vermorel et al 1987). Les besoins ont été calculés à partir du poids vif des animaux et de la production de lait $4 \%$, les apports à partir des quantités ingérées d'aliments et de leur valeur énergétique. Celle-ci a été estimée, selon les informations disponibles dans chaque publication, à partir de leur teneur en énergie digestible ou métabolisable, selon les relations proposées par Vermorel et al (1987), de la composition chimique des aliments ou des tables de la valeur nutritive (INRA 1988). Ces apports ont ensuite été corrigés pour tenir compte de l'effet du niveau alimentaire et de la proportion de concentré dans la ration sur la valeur énergétique des rations (interactions digestives et métaboliques) (Vermorel et al 1987).

Les écarts de production laitière (lait $4 \%$ ) et de composition du lait (taux butyreux et taux protéique lorsqu'ils étaient disponibles) entre les lots sur(sous)-alimentés et le lot moyen ont d'autre part été calculés pour chaque essai. Ils ont été mis en liaison avec les écarts d'apports énergétiques corrigés correspondants. De cette manière, les erreurs d'estimation de la valeur des aliments n'ont qu'une influence marginale sur les résultats de la liaison entre les écarts de performances et les écarts d'apports énergétiques.

Cette liaison a été calculée par régression non linéaire selon le modèle mononucléaire simplifié : $Y=a\left(1-b^{x}\right)$, où $Y$ représente les variations de production laitière ou de taux protéique, et X les variations d'apports énergétiques corrigés. Lorsqu'il était plus approprié, le modèle linéaire : $\mathrm{Y}=\mathrm{aX}+\mathrm{b}$ a été utilisé. Les différentes équations obtenues sont précisées au tableau 3 . 
gie nette a été linéaire et un peu plus forte, mais de manière non significative, chez les multipares que chez les primipares $(+1,0$ contre $+\mathbf{0 , 7} \mathrm{kg} / \mathrm{UFL}, \mathrm{P}>\mathbf{0 , 0 5}$ ) (figure 1). En milieu de lactation, lorsque les besoins azotés étaient couverts (79 lots), cette réponse a été curvilinéaire $(1,3 \mathrm{~kg} / \mathrm{UFL}$ entre -3 et -2 UFL contre $0,4 \mathrm{~kg} / \mathrm{UFL}$ entre +2 et +3 UFL ; figure 1). Dans les essais de longue durée, cette réponse a aussi été curvilinéaire : lorsque les apports étaient supérieurs aux besoins, elle a été semblable à celle observée en milieu de lactation dans la même plage de sur-alimentation ; lorsque les apports étaient inférieurs aux besoins, cette réponse a été supérieure $(\mathrm{P}<0,01)$ à celle observée en milieu de lactation $(2,2 \mathrm{~kg} / \mathrm{UFL}$ entre -2 et -3 UFL contre 1,3 $\mathrm{kg} / \mathrm{UFL}$ ) et voisine de la réponse maximale possible qui est de $2,3 \mathrm{~kg} / \mathrm{UFL}$ (1 UFL/0,44 UFL par $\mathrm{kg}$ de lait).

En milieu de lactation, le niveau de production des animaux n'a pas eu d'effet sur la réponse de production laitière aux variations d'apports énergétiques. Dans les essais de longue durée, cette réponse a été légèrement plus élevée, mais de manière non significative, chez les vaches fortes productrices que chez les vaches faibles productrices $(1,1$ contre $0,9 \mathrm{~kg} /$ UFL entre -2 et +2 UFL, $P>0,05)$.

Figure 1. Réponse de la production laitière $(d L A, k g / j)$ et du taux protéique (dTP, $g / \mathrm{kg}$ ) aux variations des apports énergétiques (dUF, UFL/j), dans les différents types d'essais.

(1) --+ : primipares; - $\cdot$ : multipares

(2) pour la production laitière

.... : essais où les besoins azotés étaient toujours couverts

++ : essais où les besoins azotés n'étaient pas toujours couverts

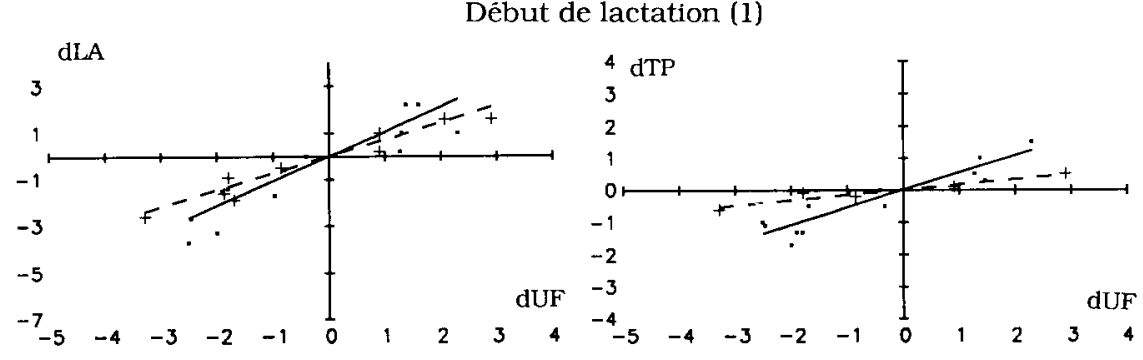

Milieu de lactation (2)

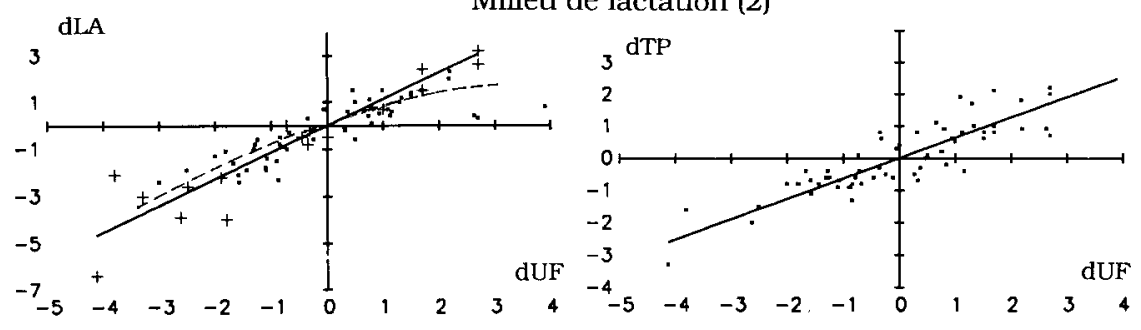

Longue durée

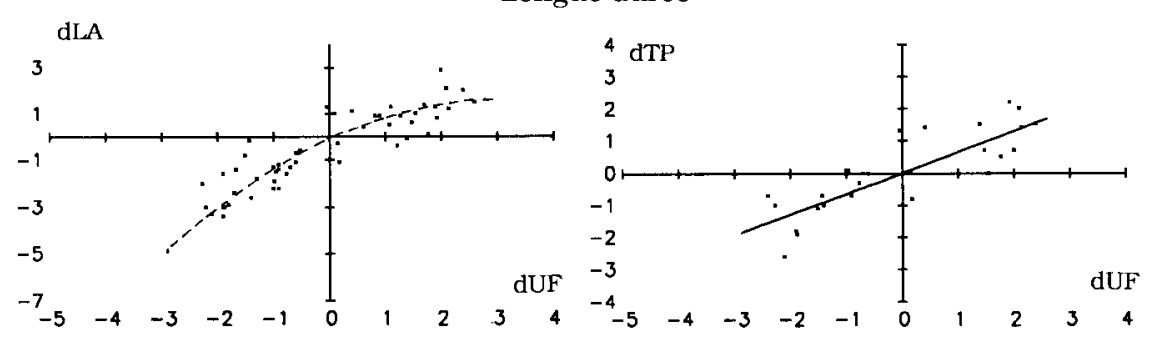

Lorsque les apports azotés variaient conjointement aux apports énergétiques (analyse réalisée sur 26 lots expérimentaux en milieu de lactation, cf origine des données), la réponse de production laitière à ces apports a été linéaire et plus élevée $(P<0,01)$ que dans les essais où les besoins azotés étaient constamment couverts (figure 1): en particulier, la production laitière a continué d'augmenter fortement $(+1,1 \mathrm{~kg} / \mathrm{UFL})$ au delà de la couverture des besoins énergétiques.

Figure 2. Réponse du taux butyreux (dTB, $g / \mathrm{kg}$ ) aux variations des apports énergétiques (dUF, $U F L / j)$.

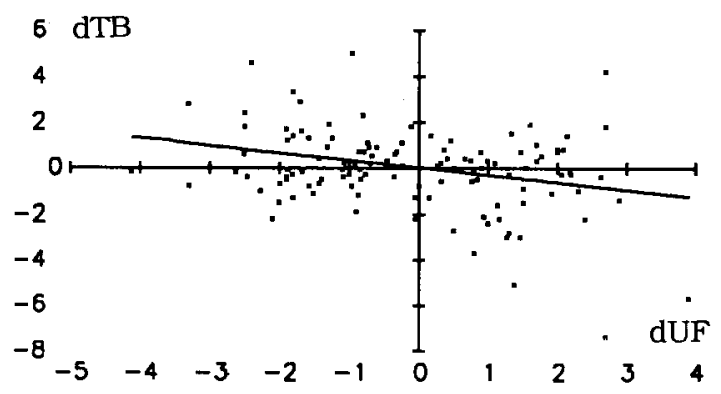

\section{2 / Variations de la composition chimique du lait}

La réponse du taux protéique aux apports énergétiques a été linéaire dans les trois groupes d'essais (D, M et L) analysés (figure 1). En début de lactation, elle a été plus élevée $(\mathrm{P}<0,01)$ chez les multipares $(0,5 \mathrm{~g}$ p.1000/ UFL) que chez les primipares (0,2 g p.1000/ UFL). En moyenne, cette réponse a été 2 fois plus faible en début de lactation $(0,3 \mathrm{~g}$ p. 1000/ UFL) qu'en milieu de lactation ou qu'au cours des essais de longue durée $(0,6 \mathrm{~g}$ p.1000/UFL). Cette réponse, mesurée en milieu de lactation, a été significativement plus élevée $(\mathrm{P}<0,05)$ chez les faibles productrices $(0,8 \mathrm{~g} \mathrm{p.1000/UFL)}$ que chez les fortes productrices $(0,4 \mathrm{~g} \mathrm{p} .1000)$ UFL) ; à l'inverse, dans les essais de longue durée, elle a été supérieure chez les vaches fortes productrices $(1,0 \mathrm{~g}$ p.1000/UFL contre 0,5 g p.1000/UFL, $\mathrm{P}<0,01$ ).

Les variations du taux butyreux en fonction du niveau des apports énergétiques ont été relativement faibles : en moyenne, l'augmentation de ces apports s'accompagne d'une diminution du taux butyreux de $0,3 \mathrm{~g}$ p. $1000 / \mathrm{UFL}$ $(R=-0,30, P<0,01)$, voisine dans les 3 types d'essais (D, M et L), mais avec des variations très importantes d'un essai à l'autre (figure 2) (écart-type résiduel de la régression de $1,7 \mathrm{~g}$ p. 1000).

\section{3 / Liaisons entre les variations de la production et de la composition du lait}

Les variations du taux butyreux ont été pratiquement indépendantes de celles de la production laitière et du taux protéique. 
En début de lactation, les variations du taux protéique sous l'effet du niveau des apports énergétiques ont été faibles comparativement à celles de la production laitière (le taux protéique augmente de 0,6 g p.1000 pour $1 \mathrm{~kg} / \mathrm{j}$ d'augmentation de la production laitière). Cette augmentation du taux protéique est un peu plus importante dans les essais de longue durée (0,8 g p.1000 pour $1 \mathrm{~kg} / \mathrm{j}$ d'augmentation de la production laitière). En milieu de lactation, la relation entre les variations du taux protéique et de la production laitière a été curvilinéaire: en dessous de la couverture des besoins énergétiques, la diminution du taux protéique est faible comparativement à celle de la production laitière $(-0,5 \mathrm{~g}$ p.1000 par $\mathrm{kg}$ de lait en moins), alors qu'au dessus des besoins l'augmentation du taux protéique est en moyenne importante $(1,8 \mathrm{~g}$ p. 1000 par $\mathrm{kg}$ de lait en plus), mais très variable d'un essai à l'autre (figure 3). Cette variabilité est due en partie aux différences de niveau d'apport azoté d'un lot à l'autre. Dans la majorité des lots où l'augmentation des apports énergétiques s'accompagnait d'une augmentation des apports azotés (essais où les besoins azotés n'étaient pas toujours couverts), un accroissement important a été observé à la fois sur la production laitière et sur le taux protéique. Dans les essais où les besoins azotés étaient toujours couverts, cet accroissement porte essentiellement sur le taux protéique.

Figure 3. Variations de la production laitière ( $d L A$, $\mathrm{kg} / \mathrm{j}$ ) en fonction de celles du taux protéique (dTP, $\mathrm{g} / \mathrm{kg}$ ) dans les essais réalisés en milieu de lactation.

- : essais où les besoins azotés étaient toujours couverts :

+ essais oú les besoins azotés n'étaient pas toujours couverts.

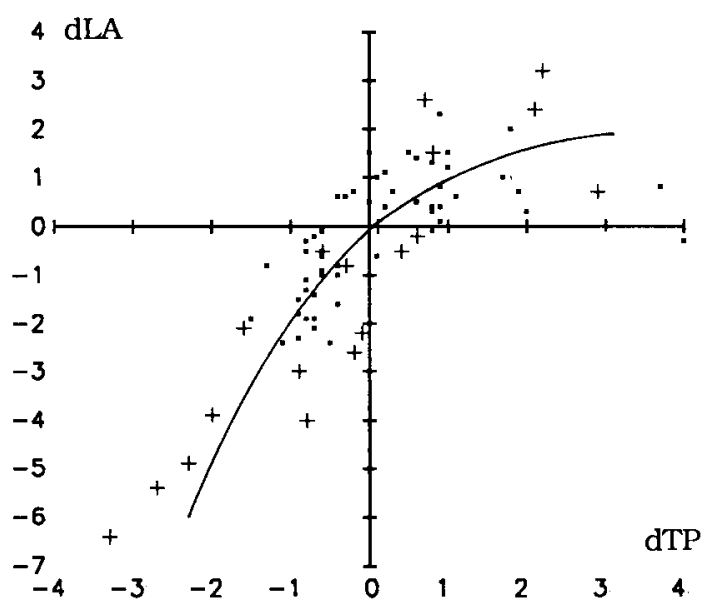

\section{3 / Discussion et conclusions}

Les réponses de production laitière obtenues dans cette étude sont légèrement supérieures à la plupart de celles observées dans d'autres études bibliographiques, en raison de la correction des apports énergétiques pour tenir compte des phénomènes d'interactions digestives et métaboliques. Calculés sans faire cette correction, ils s'inscrivent tout à fait dans la
Figure 4. Influence des variations d'apports énergétiques par rapport à la satisfaction des besoins (dUF, UFL/j) sur la production laitière ( $d L A, k g / j)$ et le taux protéique (dTP, $\mathrm{g} / \mathrm{kg}$ ) dans les différents types d'essais.

(1) Déficit énergétique accepté en début de lactation sur une vache produisant $25 \mathrm{~kg} / \mathrm{j}$ de lait $4 \%$ (Faverdin et al, 1987).

-..- : essais en début de lactation (multipares)

$-:$ essais en milieu de lactation (besoins azotés couverts)

-- : essais de longue durée.
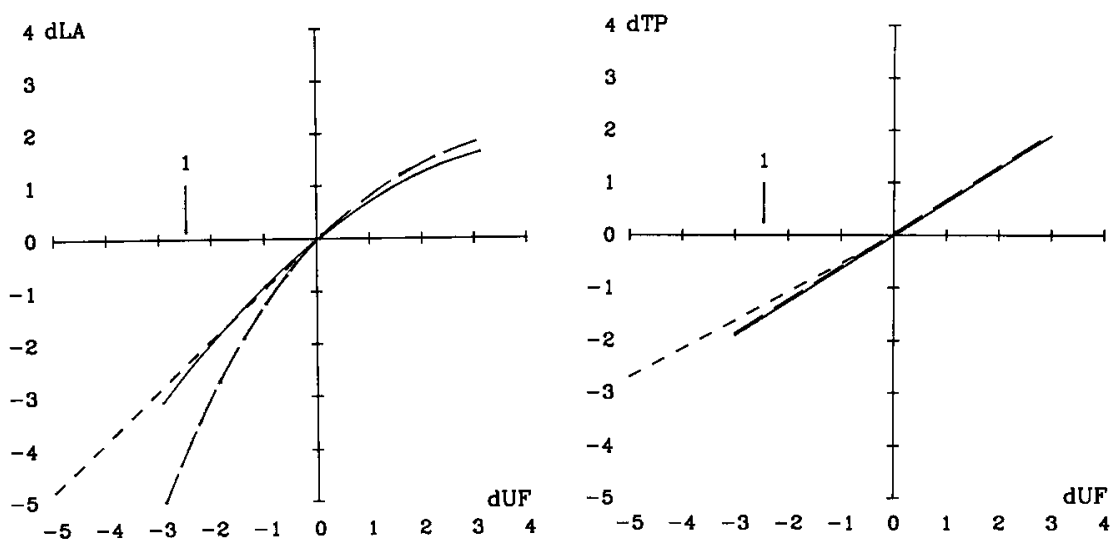

gamme des valeurs moyennes proposées par Ekern (1972), Wiktorsson (1979) ou Rémond (1985).

L'utilisation de l'énergie métabolisable pour la production laitière est maintenant bien connue (environ 0,60 ) et constante sur une large plage de production, qu'il s'agisse de variations entre animaux de potentiels de production différents, ou d'animaux recevant des niveaux d'alimentation différents (Flatt et al 1972, Van Es 1975). Les rendements marginaux toujours inférieurs au rendement maximal théorique $(2,3 \mathrm{~kg}$ de lait à $4 \%$ de matières grasses/UFL) que nous avons observés résultent donc de l'effet de la variation de la quantité d'énergie apportée aux animaux, à la fois sur la sécrétion de lait et sur la quantité de réserves corporelles.

Dans ces conditions, cette étude a permis de confirmer et de préciser les résultats obtenus ou les hypothèses émises par différents auteurs. En début de lactation, la réponse de la production laitière est en moyenne supérieure à celle observée en milieu de lactation, comme cela a été couramment observé (Broster et Broster 1984). Cela s'explique d'abord par le fait que le niveau de couverture des besoins énergétiques à cette période n'est pratiquement jamais réalisé. La réponse observée chez les multipares en début de lactation est ainsi voisine de celle observée en milieu de lactation pour un déficit énergétique de 2 UFL (figure 4), ce qui correspond à peu près au déficit moyen observé dans les lots «moyens » en début de lactation (tableau 2). D'autre part, il est possible que la concentration en MAT des rations distribuées dans les essais en début de lactation, bien que plus élevée que dans les essais en milieu de lactation, n'ait pas été suffisante à une période où la réponse de production aux apports azotés est importante (Vérité 1983). Le fait que cette réponse soit linéaire est certainement dû en partie à la plage de variation des données (Broster et al 1985), plus restreinte en début de lactation qu'en milieu de lactation ou que dans les
La réponse du taux protéique du lait à des variations d'apports énergétiques est linéaire en début qu'en milieu de lactation. mais plus faible 
essais de longue durée. D'ailleurs, pour ces 2 périodes, la réduction de cette plage de variation rend la relation pratiquement linéaire.

La réponse de production laitière supérieure sur les essais de longue durée comparativement aux essais de courte durée, surtout lorsque le niveau des apports est éloigné des besoins, confirme les observations de Broster et Broster (1984). Cela signifie que le rôle tampon joué par les réserves corporelles diminue avec le temps, en raison de leur épuisement progressif et, corrélativement, d'une régulation du métabolisme de l'animal qui restreint ses dépenses (lait) afin de maintenir son homéostasie. Cette situation est généralement plus rapidement atteinte chez les vaches fortes productrices (dont l'état corporel est souvent inférieur à celui de vaches plus faibles productrices), ce qui expliquerait la tendance observée dans cette étude à une plus forte diminution de la production à long terme chez ces animaux, et confirmerait les observations faites dans le même sens dans certaines comparaisons intraessai (Wiktorsson 1979, Kroll et al 1986, Coulon et al 1987). Les résultats concernant l'effet du niveau de production sur le rendement marginal en lait restent cependant contradictoires : d'autres auteurs ont ainsi montré l'absence d'effet de ce facteur (Wiktorsson 1971, Johnson 1979, Gordon 1984). Cette divergence, au moins apparente, peut avoir 2 explications :

- comme le signale Leaver (1988), la réponse marginale de production laitière à un apport supplémentaire de concentré dépend de la distribution à volonté ou non du fourrage ; lorsque celui-ci est offert à volonté, les réponses marginales à l'apport de concentré sont les mêmes pour des vaches fortes ou faibles productrices, alors que lorsque le fourrage est restreint, les fortes productrices présentent des réponses marginales plus importantes (Leaver 1988) ;

- il est probable que lorsque des vaches de différents potentiels de production étaient comparées dans les mêmes essais, les vaches faibles productrices avaient tendance à être mieux ali-

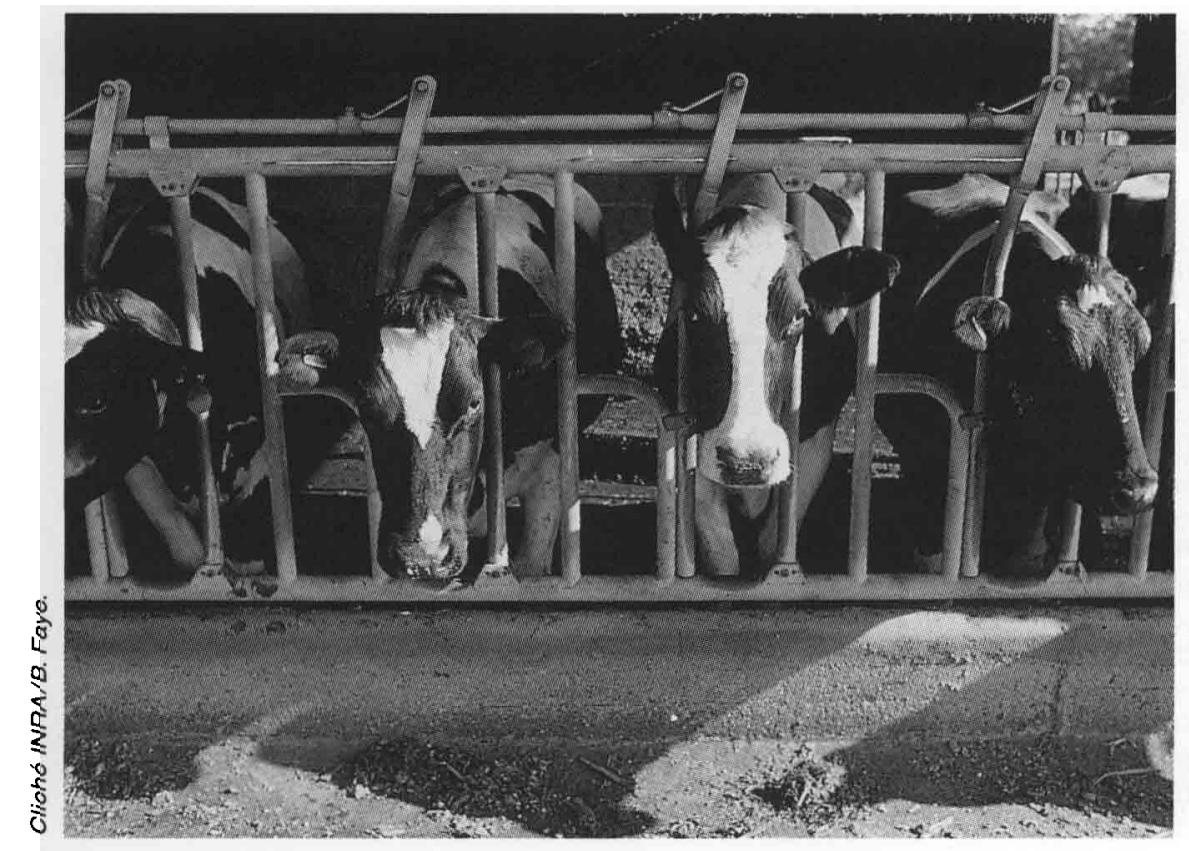

mentées relativement à leurs besoins que les vaches fortes productrices: la loi des rendements décroissants entraîne alors une réponse plus importante des vaches moins bien alimentées.

La réponse de production laitière plus élevée lorsque les apports azotés étaient faibles et s'accroissaient en même temps que les apports énergétiques pourrait être liée 1) à un accroissement des apports énergétiques supérieur à celui que nous avons calculé et qui résulterait de l'amélioration de la digestibilité de la ration (et donc de sa valeur énergétique) apportée par son enrichissement en azote. Vérité (1978) et Rémond (1985) ont en effet observé des accroissements de valeur énergétique compris entre 0,01 et 0,02 UFL par $\mathrm{kg}$ MS de ration et par point p. 100 de MAT supplémentaires jusqu'à des teneurs de $18 \%$ de MAT dans la ration, 2) à une meilleure utilisation des nutriments absorbés pour la production laitière, due soit à une amélioration de l'efficacité de la synthèse du lait, soit à un partage de l'utilisation des nutriments plus favorable à la production laitière.

La réponse de taux protéique a été linéaire sur la plage des apports énérgétiques considérée, ce qui confirme les observations de Emery (1978), Rémond (1985) et Spörndly (1989). Elle a été supérieure (sauf en début de lactation) à celle observée par ces auteurs $[0,35$ à 0,50 $\mathrm{g} / \mathrm{UFL}$ ) pour la même raison que celle évoquée pour la production laitière. Elle a été plus faible en début de lactation qu'en milieu. Il semblerait donc qu'en début de lactation l'augmentation des apports énergétiques stimule plus la production des matières protéiques, en les concentrant peu, alors que par la suite, ce serait la concentration de ces matières qui serait favorisée. Ceci est peut-être lié au niveau de couverture des besoins énergétiques en début de lactation. La relation curvilinéaire obtenue entre ces 2 paramètres en milieu de lactation semble montrer que lorsque les apports sont inférieurs aux besoins, la production laitière augmente plus que le taux protéique, alors qu'au-delà, c'est plutôt l'inverse.

En pratique, ces résultats permettent de mieux raisonner les conséquences de différentes stratégies de rationnement sur la production et la composition du lait (tableau 4). Ils mettent principalement en évidence l'importance de la durée de la période de sur-(sous)alimentation énergétique et de son association ou non à une sous-alimentation azotée.

Par ailleurs, ils semblent montrer que l'effet $\mathrm{du}$ niveau de production des animaux, s'il existe, est complexe (variable suivant la durée de la sous-alimentation). Il doit être interprété avec prudence, en raison de la liaison probable entre ce facteur et le niveau des réserves corporelles, liaison qui n'a pas pu être quantifiée dans cette étude. En effet, l'absence, dans la majorité des essais, de la notation de l'état corporel des animaux, n'a pas permis de tenir compte de ce facteur, dont on sait pourtant qu'il interagit fortement avec le niveau d'apport énergétique dans la réponse de production laitière à la sous-alimentation (Garnsworthy 1988). 
Tableau 4. Conséquences d'une sous-alimentation énergétique (par rapport à la couverture stricte des besoins) à court ou à long terme sur la production laitière et le taux protéique.

Exemple d'une vache laitière en milieu de lactation produisant $30 \mathrm{~kg} / \mathrm{j}$ de lait, recevant, à volonté, une ration de base de qualité excellente (0,9 UFL et $1,03 \mathrm{UEL} / \mathrm{kg}$ MS) ou moyenne $(0,7 \mathrm{UFL}$ et $1,1 \mathrm{UEL} / \mathrm{kg} \mathrm{MS})$, complémentée à l'aide d'un concentré à 1 UFL/kg. Dans toutes les situations les besoins azotés sont supposés couverts.

\begin{tabular}{|c|c|c|c|c|}
\hline \multirow{2}{*}{$\begin{array}{l}\text { Qualité du fourrage } \\
\text { Variation d'apport de concentré }(\mathrm{kg} / \mathrm{i}) \text { par rapport } \\
\text { à la couverture des besoins énergétiques }\end{array}$} & \multicolumn{2}{|c|}{ excellente } & \multicolumn{2}{|c|}{ moyenne } \\
\hline & -2 & -3 & -2 & -3 \\
\hline \multicolumn{5}{|l|}{ Variation de la production laitière $(\mathrm{kg} / \mathrm{j})$} \\
\hline - à court terme $(<3$ mois $)$ & $-0,7$ & $-1,3$ & $-1,1$ & $-1,8$ \\
\hline - à long terme ( $>3$ mois $)$ & $-1,0$ & $-1,9$ & $-1,6$ & $-2,6$ \\
\hline Variation du taux protéique $(\mathrm{g} / \mathrm{kg})$ & $-0,6$ & $-1,0$ & $-0,9$ & $-1,2$ \\
\hline
\end{tabular}

\section{Références bibliographiques}

BROSTER W.H., THOMAS C., 1981. The influence of level and pattern of concentrate input on milk output. In "Recent advances in animal nutrition ». pp 49-69. Ed W. Haresign.

BROSTER WH. SUTTON JD BINES IA BROSTER V.J., SMITH T., SIVITER J.W., JOHNSON V.W. NAPPER D.J., SCHULLER E., 1985. The influence of plane of nutrition and diet composition on the performance of dairy cows. J. Agric. Sci., 104, 535-557.

BROSTER W.H., BROSTER V.J., 1984. Reviews of the progress of dairy science : long term effects of plane of nutrition on the performance of the dairy cow. J. Dairy Res. $, 51,149-196$

COULON J.B., PETIT M., D'HOUR P., GAREL J.P., 1987. The effect of level and distribution of concentrate supplementation on performance of dairy cows. Livest. Prod. Sci., 17, 117-133.

FAVERDIN P., HODEN A., COULON J.B., 1987. Recommandations alimentaires pour les vaches laitières. Bull. Tech. CRZV Theix, INRA, 70, 133-152.

DOREAU M., REMOND B., 1983. Capacité digestive et niveau de production laitière. Bull. Tech. CRZV Theix, INRA, 53, 17-26.

DULPHY J.P., FAVERDIN P., MICOL D., BOCQUIER F., 1987. Révision du système des Unités d'Encombrement (UE). Bull. Tech. CRZV Theix, INRA, 70,35-48.

EMERY R.S., 1978. Feeding for increased milk protein. J. Dairy Sci., 61, 825-828.

FLATT W.P., MOE W.P., MOORE L.A., BREIREM K., EKERN A., 1972. Energy requirements in lactation. In Handbuch der tierenahrung. Ed W. Lenkeif and K. Breirem. Verlag Paul PAREY Hambourg and Berlin. pp 341392.

GARNSWORTHY P.C., 1988. The effect of energy reserves at calving on performance of dairy cows. In Nutrition and lactation in the dairy cow. Ed P.C. Garnsworthy, Butterworth, London. pp 157-170.

GORDON F.J., 1984. The effect of level of concentrate supplementation given with grass silage during the winter on the total lactation performance of autumn-calving dairy cows. J. Agric. Sci, 102, 163-179.

GORDON F.J., 1980. Feed input-milk output relationships in the spring calving dairy cow. In Recent advances in animal nutrition. Ed W. Haresign, Butterworth, London, pp 15-31.

INRA, 1988. Alimentation des bovins, ovins et caprins. ED R. Jarrige, INRA Publications.
JONES G.P., GARNSWORTHY P.C., 1989. The effects of dietary energy content on the response of dairy cows to body condition at calving. Anim. Prod., 49, 183-191.

KROLL O., OWEN J.B., WHITAKER , 1987. Grouping and complete diet composition in relation to parity and potential yield in dairy cows. J. Agric. Sci., 108, 281-291. LARNICOL S.. 1984. Etat d'engraissement des vaches laitières à la mise bas : effet sur quelques paramètres zootechniques selon le niveau d'alimentation en début de lactation. Thèse docteur ingénieur, ENSA Montpellier. 103 pp.

LEAVER J.D., 1988. Level and pattern of concentrate allocation to dairy cows. In Nutrition and lactation in the dairy cow. Ed P.C. Garnsworthy, Butterworth, London. pp $315-326$.

REMOND B., 1985. Influence de l'alimentation sur la composition du lait de vache 2. Taux protéique: facteurs généraux. Bull. Tech. CRZV Theix, INRA, 62, 53-67.

SPÖRNDLY E., 1989. Effects of diets on milk composition and yield of dairy cows with special emphasis on milk protein content. Swedish J. Agric. Res., 19, 99-106.

SUTTON J.D., 1989. Altering milk composition by feeding. J. Dairy Sci., 72, 2801-2814.

VERITE R., 1983. Particularités de la nutrition azotée. Bull. Techn. CRZV Theix, INRA, 53, 65-74.

VERITE R., PEYRAUD J.L., 1989. Protein : the PDI systems. In Ruminant Nutrition : recommended allowances and feed tables. Ed R. Jarrige, John Libbey Eurotext. pp 33-48.

VERMOREL M., COULON J.B., JOURNET M., 1987. Révision du système des unités fourragères (UF). Bull. Tech. CRZV Theix, INRA, 70, 9-18.

WIKTORSSON H., 1971. Studies on the effects of different levels of nutrition to dairy cows. Swedish J. Agric. Res., 1, 83-103.

WIKTORSSON H., 1979. General plane of nutrition for dairy cows. In Feeding strategy for the high yielding dairy cow. Ed W.H. Broster and H. Swan, St Albans, Granada. pp 149-169.

La version initiale de ce texte, en langue anglaise, est en cours de publication dans la revue Livestock Production Science. 


\section{Summary}

Variations in milk output and 'milk fat and protein content in response to the level of energy supply to the dairy cow : a review.

The purpose of this review was to determine with accuracy the effect of the lactation stage, length of under/over-feeding, age and production potential of the animals on the milk output and milk protein content response to variations in the energy supply. Sixty-six feeding trials on dairy cows producing 9 to $29 \mathrm{~kg} / \mathrm{d}$ (6 to 30 cows/experimental group) and including at least 3 levels of energy supply were compared. The energy supply was expressed as an energy unit effectively available to the animal (Feed Unit for Lactation, UFL). In early lactation, the milk yield response to energy supply was linear and large $(+0.9 \mathrm{~kg}$ of milk/UFL). In mid-lactation and in long-term trials this response was curvilinear. When the supply was below the requirements, the response was higher in long-term trials than in short-term trials performed in mid-lactation $(2.2 \mathrm{~kg} / \mathrm{UFL}$ versus $1.3 \mathrm{~kg} / \mathrm{UFL}, \mathrm{P}<\mathbf{0 . 0 1}$ ). The protein content response to energy supply was linear whatever the stage of lactation or the length of the trials. In early lactation, it was half that in mid-lactation or in long-term trials $(0.6 \mathrm{~g}$ p. 1000/UFL). The relation between variations in milk yield and in milk protein content was curvilinear and depended on the stage of lactation and on the level of feeding proportional to the energy requirements. It was not possible to demonstrate any significant effect of the production potential on milk yield and milk protein content responses to variations in energy supply.

COULON J.B., REMOND B., 1991. Réponses de la production et de la composition du lait de vache aux variations d'apports nutritifs. INRA Prod. Anim., 4 (1), $49-56$. 